\title{
Diet and nutrition support after a pelvic cancer diagnosis: a cross-sectional study.
}

\author{
Georgios Saltaouras, Eila Watson, Shelly Coe and Helen Lightowler \\ Oxford Brookes University, Oxford, United Kingdom
}

\section{Abstract}

A cancer diagnosis serves as a teachable moment for patients to consider lifestyle changes to cope with treatment and for future health. Diet and nutrition is an important, yet neglected aspect of survivorship care. Patients diagnosed with a cancer in the pelvis (anal, bladder, rectal and cancers of the reproductive organs) may benefit from dietary modifications that could improve quality of life and may influence survival. This project aims to explore pelvic cancer patients' dietary habits and experiences of nutrition support in cancer care.

People who have been diagnosed with a pelvic cancer and either undergoing $(n=266)$ or having completed radiotherapy treatment 6-24 months previously $(n=405)$ were invited to fill in a survey about their current dietary habits and nutritional support received after diagnosis. Data were analysed using descriptive statistics and logistic regression.

Respondents $[n=251$; mean (SD) age $68.8(10.5)$ years] reported high obesity rates $(63 \%$ classified as overweight or obese) and presence of treatment side effects $(82 \%)$ and comorbidities $(75 \%)$. There was no significant difference in obesity rates, presence of side effects or comorbidities between on-treatment and post-treatment patients $(P>0.01$ for all comparisons). Two thirds of respondents $(n=170)$ reported at least one dietary change since diagnosis and 31\% $(n=77)$ reported supplement use. However, the number of respondents who reported a change per food group was low. Most notable changes included increased intake of vegetables (33\%), fruit $(33 \%)$ and oily fish $(29 \%)$ and reduction of sugary foods $(48 \%)$, alcohol $(41 \%)$ and processed meats $(40 \%)$. Forty-three percent reported receipt of dietary advice from the healthcare team; mainly leaflets from cancer specialist nurses. Receipt of dietary support from the healthcare team was a significant predictor of dietary change (OR 4.02, 95\% CI: 2.01-8.05). Thirty-two percent of respondents $(n=79)$ reported looking for advice from other sources, with the internet being the most popular $(66 \%$ of them looked at medical and/or charity websites). Survivors were interested in receiving dietary support in relation to weight management, management of side effects and future health; however, they were uncertain of the benefits of diet in improving low appetite and the feeling of fatigue.

Results from this study highlight an increased need for dietary support in pelvic cancer populations post-treatment. Emphasis should be given to the role of the healthcare team in the delivery of nutritional information to influence patients make healthy dietary choices.

\section{Conflict of Interest}

There is no conflict of interest. 